\title{
Compact, Low-Loss Waveguide Crossings for High-Index-Contrast SOI Photonic Wires
}

\author{
Wim Bogaerts, Pieter Dumon, Dries Van Thourhout, Roel Baets \\ Ghent University - Interuniversity Microelectronics Center (IMEC), Department of Information Technology \\ Sint-Pietersniewstraat 41, 9000 Gent, Belgium \\ Wim.bogaerts@intec.ugent.be
}

Abstract: Waveguide crossings are essential for complex photonic circuits. We present compact crossings for silicon-on-insulator photonic wires. By locally applying a lower index contrast, $97.5 \%$ transmission is achieved in a $6 \mu \mathrm{m}$ length with only $-40 \mathrm{~dB}$ crosstalk. (C2006 Optical Society of America

OCIS codes: (130.0130) Integrated Optics; (130.1750) Components, (130.2790) Guided Waves

\section{Introduction}

Silicon-on-insulator (SOI) nanophotonic waveguides make it possible to combine many compact functions onto a single photonic integrated circuit [1]. Because of the high refractive index contrast (3.45 to 1.45), tightly confined waveguides with submicron core sizes become possible. Moreover, these very compact photonic wires can be fabricated with commercial CMOS fabrication technology, allowing low-cost large-scale manufacturing. While current propagation losses of about $2.5 \mathrm{~dB} / \mathrm{cm}$ [1][9] allow already centimetres-long on-chip waveguides, integrating many functions on a planar circuit is often limited by the topology of the interconnections between building blocks. While in electronics many-layered interconnects allow flexible routing of circuits, current SOI photonic chips are limited to single-layered circuits. This makes the routing of more complex photonic circuits difficult without using direct waveguide crossings.

A direct waveguide crossing causes a local loss of lateral optical confinement, causing diffraction of the waveguide. In low-contrast waveguides direct waveguide crossings only present a minor perturbation of the straight waveguide. Even though the core (and therefore the length of the crossing) can be several microns in size, the beam is hardly diffracted in a crossing. This is not true in high-contrast systems like SOI. Guided modes in photonic wires contain spatial components at large angles with the propagation direction. At a direct crossing, the mode is strongly diffracted and a sizeable fraction of the light cannot be recaptured by the waveguide core at the other side. Calculations indicate losses in the order of $-1.4 \mathrm{~dB}$ (72\% transmission) per crossing [2], and this depends strongly on the wire width. Also, this generates significant crosstalk of approximately $-9 \mathrm{~dB}$ in the arms of the crossing.

We present a technique which improves the transmission of the crossing by expanding the waveguide mode and locally reducing the refractive index contrast. At the same time, this also reduces the crosstalk.

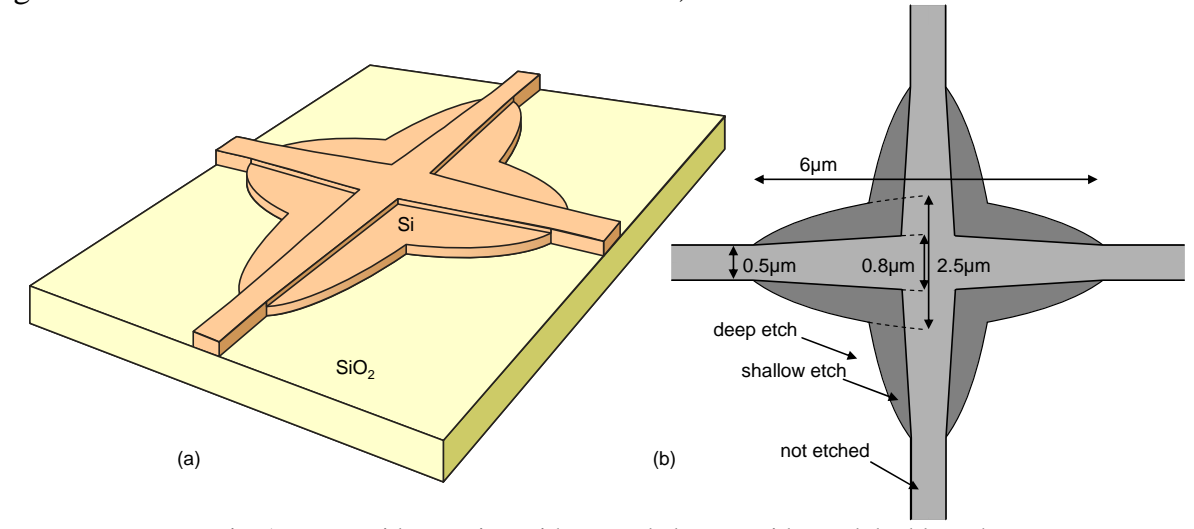

Fig. 1. Waveguide crossing with expanded waveguides and double etch.

\section{Design}

One can improve the transmission by either enhancing the direct coupling to the other waveguide by reducing diffraction [2][3], or design a cavity which resonates in such a way to enhance the coupling to the correct waveguide [4]. However, the latter approach implies a strong wavelength dependence. We try to suppress the diffraction at the crossing by reducing the wide-angle components of the mode through a broadening of the waveguide core, e.g. by using elliptical mode expanders as shown in Fig. 1b-c [2]. However, in a four-fold symmetric crossing, as in Fig. 1c, expanding the core also increases the length of the crossing, causing more severe loss of confinement and wider diffraction. This is already shown in [2], where a four-fold symmetric crossings has a calculated loss of $-0.4 \mathrm{~dB}$, 


\section{OTuM1.pdf}

while a crossing with only one expanded waveguide has only - $0.1 \mathrm{~dB}$ loss for the expanded waveguide (the losses are much higher for the narrow arms, making this unsuitable for actual waveguiding). Still, these designs require crossing lengths of at least $10 \mu \mathrm{m}$.

To reduce the diffraction, we propose a scheme to maintain confinement over a larger part of the expanded crossing, while at the same time lowering the lateral refractive index contrast. This reduces the diffraction in the non-confined region. To reduce the lateral index contrast of the waveguide, we use a double-etch scheme [1][6]. This is illustrated in Fig. 1. While the high-contrast photonic wires and the mode expanders are defined by completely etching the silicon core, a second contrast level is defined by a shallow etch, confining the light to the center of the mode expander. Also, by lowering the average refractive index in the outer part of the crossing, the diffraction and curving of the phase fronts is partially compensated. In contrast with [2], we use a parabolic instead of an elliptical mode expander. This shape is preferable as this suppresses the excitation of higher-order modes [5].

This is made clear in the simulation results from Fig. 2. We used 2-D eigenmode expansion for a wavelength of $1.55 \mu \mathrm{m}$. The direct crossing (a) is the most compact, but has only $67 \%$ transmission, and more important, several percent of crosstalk in the arms. This is in good agreement with [2]. The two-fold symmetric elliptical crossing (b) was modelled after the results presented in [2], but with only $6 \mu \mathrm{m}$ length. This reduction in length reduces the transmission to $61 \%$. This effect is even more dramatic for the four-fold symmetric elliptical crossing (c), which has only $32 \%$ transmission. The loss of confinement in the crossing region is very severe. By using the double etch scheme (d) this loss of confinement is largely compensated, boosting the transmission to more than 95\%.

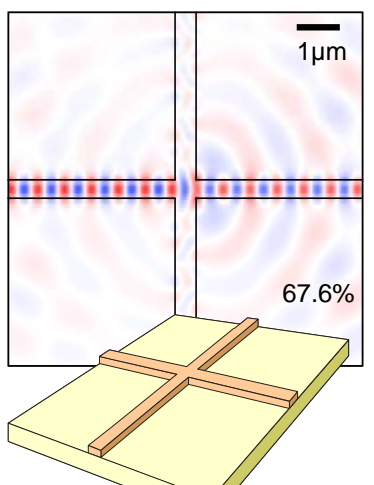

(a)

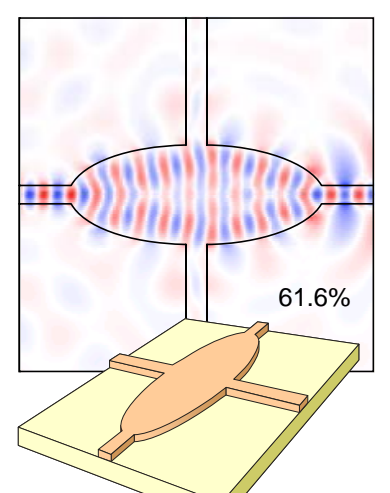

(b)

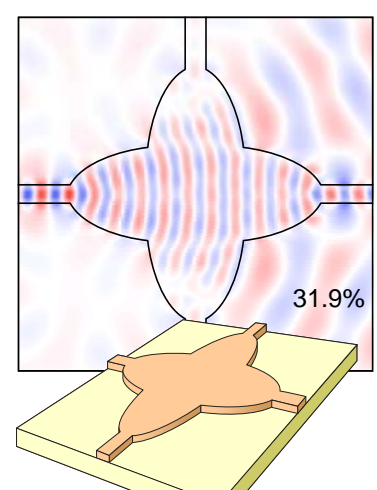

(c)

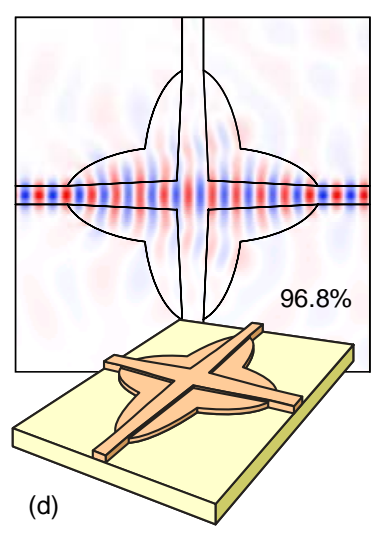

(d)

Fig. 2. 2-D simulations of different waveguide crossings. (a) direct crossing, (b) two-fold symmetric elliptical crossing as presented in [2], but with a length of $6 \mu \mathrm{m}$, (c) elliptical crossing from [2], but with a $6 \mu \mathrm{m}$ length, (d) crossing with deep and shallow etch.

\section{Implementation of a low-contrast waveguide crossing}

The waveguides and couplers were fabricated in commercial 200mm SOI wafers with a top silicon layer of $220 \mathrm{~nm}$ and a buried oxide of $2 \mu \mathrm{m}$. First, the shallow-etched structures are defined using deep UV lithography at 248nm and dry etching. Subsequently, the deeply etched waveguides are fabricated in a similar way [1]. Alignment between the two process layers is maintained by the lithography tool with an accuracy of approximately 50nm. The fabricated device is shown in Fig. 3.

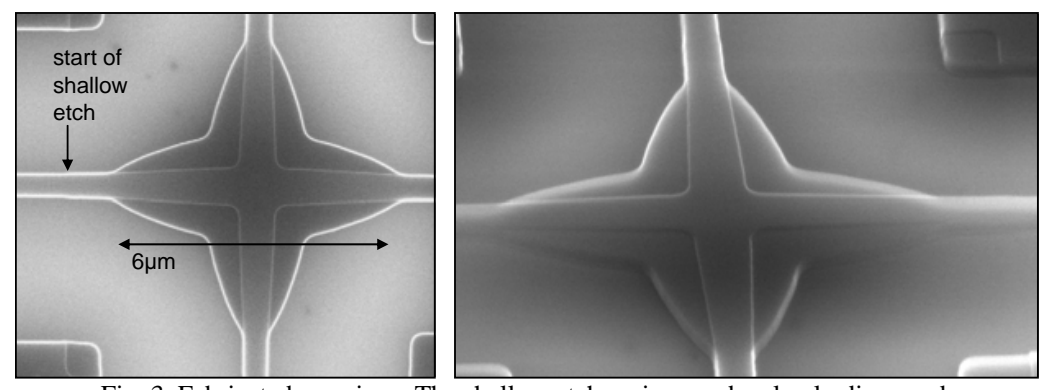

Fig. 3. Fabricated crossings. The shallow etch region can be clearly discerned.

The crossing has a length of $6 \mu \mathrm{m}$ and the parabolic expander would reach a center width of $2.5 \mu \mathrm{m}$ (if no perpendicular crossing arm would be defined) as shown in Fig. 1b. The shallow etched waveguide also slightly expands from 500nm to 800nm over the length of the crossing. 


\section{OTuM1.pdf}

For characterization, we used vertical grating fiber couplers [1][7]. We measured waveguides with 0, 5, 10 and 21 crossings. The transmission characteristics as a function of wavelength are shown in Fig. 4a. The Gaussian shape of the curves is characteristic for the grating fiber coupler gratings and is not a feature of the crossings [7]. In Fig. 4b we extract the transmission per crossing using a fit on the measurements at a wavelength of $1550 \mathrm{~nm}$. The loss per crossing is $-0.16 \mathrm{~dB}$, which amounts to $97.5 \%$ transmission. We also extracted an upper value for the crosstalk by measuring the output from the side arm of the first crossing. We found the crosstalk to be better than $-40 \mathrm{~dB}$. We also repeated the measurements for different waveguide widths, and found the performance to be the same within the error margin of our measurements. This indicates that the crossings are quite tolerant to fabrication variations.
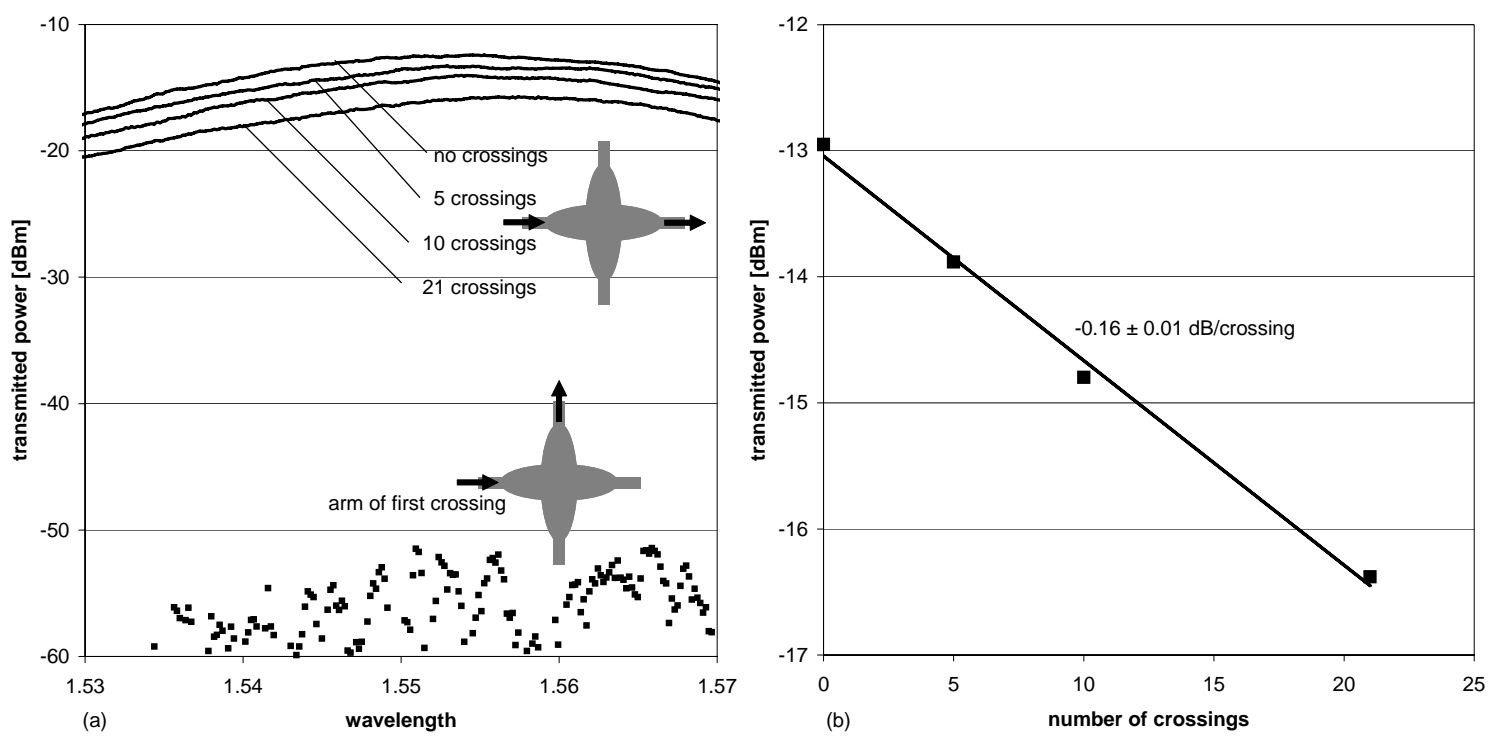

Fig. 4. Transmisison measurements on multiple waveguide crossings. (a) transmission spectrum for 0, 5, 10 and 21 crossings. The parabolic Gaussian spectrum is characteristic for grating fiber couplers [7]. (b) fitted loss per crossing at a wavelength of 1550nm.

\section{Conclusion}

We've demonstrated compact, low-loss crossings for SOI photonic wire waveguides. The crossings are implemented using parabolically broadened waveguides and a double etch scheme to reduce lateral refractive index contrast. We measured $-0.16 \mathrm{~dB}$ loss and $-40 \mathrm{~dB}$ crosstalk.

\section{Acknowledgements}

This work was partly supported by the European Union through the IST-PICMOS project, the IST-ePIXnet Network of Excellence and the Silicon Photonics Platform, and partly supported by the Belgian IAP-PHOTON project.

\section{References}

[1] W. Bogaerts, R. Baets, P. Dumon, V. Wiaux, S. Beckx, D. Taillaert, B. Luyssaert, J. Van Campenhout, P. Bienstman, and D. Van Thourhout, "Nanophotonic waveguides in Silicon-on-insulator fabricated with CMOS technology,” J. Lightwave Technol., vol. 23, no. 1, pp. 401-412, 2005.

[2] T. Fukazawa, T. Hirano, F. Ohno, T. Baba, “Low loss intersection of Si photonic wire waveguides”, Jap. J. Appl. Phys., vol. 43(2), p. 646647, 2004

[3] C. Wei, F. Groen, M.K. Smit, I. Moerman, P. Van Daele, R. Baets, "Intregrated Optical Elliptical Couplers: Modeling, Design, and Applications”, J. Lightwave Technol. Vol. 15 (5), p.906, 1997

[4] S.G. Johnson, C. Manolatou, S. Fan, P.R. Villeneuve, J.D. Joannopoulos, H.A. Haus, "Elimination of cross talk in waveguide intersections”, Optics Letters, vol. 23(23), p. 1855, 1998

[5] W.K. Burns, A.F. Milton, A.B. Lee, “Optical-waveguide parabolic coupling horns”, Appl. Phys. Lett., vol. 30(1), p.28-30, 1977

[6] W. Bogaerts, V. Wiaux, D. Taillaert, S. Beckx, B. Luyssaert, P. Bienstman, and R. Baets, "Fabrication of photonic crystals in Silicon-oninsulator using 248-nm deep UV lithography,” IEEE J. Sel. Topics. Quant. Electron., vol. 8, no. 4, pp. 928-934, 2002.

[7] D. Taillaert, W. Bogaerts, P. Bienstman, T. Krauss, P. Van Daele, I. Moerman, S. Verstuyft, K. De Mesel, and R. Baets, “An out-of-plane grating coupler for efficient butt-coupling between compact planar waveguides and single-mode fibers,” IEEE J. Quant. Electron., vol. 38, no. 7, pp. 949-955, 2002.

[8] W. Bogaerts, D. Taillaert, B. Luyssaert, P. Dumon, J. Van Campenhout, P. Bienstman, D. Van Thourhout, R. Baets, V. Wiaux, and S. Beckx, "Basic structures for photonic integrated circuits in Silicon-on-insulator," Opt. Expr., vol. 12, no. 8, pp. 1583-1591, April 2004.

[9] P. Dumon, W. Bogaerts, V. Wiaux, J. Wouters, S. Beckx, J. Van Campenhout, D. Taillaert, B. Luyssaert, P. Bienstman, D. Van Thourhout, and R. Baets, "Low-loss SOI photonic wires and ring resonators fabricated with deep UV lithography," IEEE Photon. Technol. Lett., vol. 16, no. 5, pp. 1328-1330, May 2004. 